\title{
Alpha2,3-sialyltransferase III knockdown sensitized ovarian cancer cells to cisplatin-induced apoptosis
}

\author{
Xiaoyu Wang ${ }^{\bowtie 1}$, Yiting Zhang ${ }^{1}$, Haiyingjie Lin $^{3}$, Yan Liu ${ }^{2}$, , Yi Tan ${ }^{2}$, Jie Lin², Fenze Gao ${ }^{4}$, Shaoqiang Lin ${ }^{\bowtie 2}$
}

1. Department of Obstetrics and Gynecology, The First Affiliated Hospital of Jinan University, 510630 Guangzhou, China

2. Central Laboratory of the First Affiliated Hospital of Jinan University, 510630 Guangzhou, China

3. College of Basic Medical Science, Dalian Medical University, 116044 Dalian China

4. School of Medicine, Jinan University, 510630 Guangzhou China

${ }^{\circledR}$ Corresponding author: Xiaoyu Wang, E-mail: twxy@jnu.edu.cn; ShaoqiangLin, E-mail: shaotsiang@163.com;

\begin{abstract}
Emerging evidence indicates that $\beta$-galactoside- $\alpha 2,3$-sialyltransferase III (ST3Gal3) involves in development, inflammation, neoplastic transformation, and metastasis. However, the role of ST3Gal3 in regulating cancer chemoresistance remains elusive. Herein, we investigated the functional effects of ST3Gal3 in cisplatin-resistant ovarian cancer cells. We found that the levels of ST3Gal3 mRNA differed significantly among ovarian cancer cell lines. H08910PM cells that have high invasive and metastatic capacity express elevated ST3Gal3 mRNA and are resistant to cisplatin, comparing to SKOV3 cells that have a lower level of ST3Gal3 expression and are more chemosensitive to cisplatin. We found that the expression of ST3Gal3 has reverse correlation with the dosage of cisplatin used in both SKOV3 and HO8910PM cells, and high dose of cisplatin could down-regulate ST3Gal3 expression. We then examined the functional effects of ST3Gal3 knockdown in cancer cell lines using FACS analysis. The number of apoptotic cells was much higher in cells if ST3Gal3 expression was knocked down by siRNA and/or by treating cells with higher dosage of cisplatin in comparison to control cells. Interestingly, in HO8910PM cells with ST3Gal3 knockdown, the levels of caspase 8 and caspase 3 proteins increased, which was more obvious in cells treated with both ST3Gal3 knockdown and cisplatin, suggesting that ST3Gal3 knockdown synergistically enhanced cisplatin-induced apoptosis in ovarian cancer cells. Taken together, these results uncover an alternative mechanism of cisplatin-resistance through ST3Gal3 and open a window for effective prevention of chemoresistance and relapse of ovarian cancer by targeting ST3Gal3.
\end{abstract}

Keywords: ST3Gal3; cisplatin; ovarian cancer; chemosensitivity

\section{Introduction}

Ovarian cancer is a widespread, pro-metastasis and lethal malignancy [1-3]. Risk factors that contribute to ovarian carcinogenesis mainly include aberrant ovulation, obesity, and genetic mutations as seen in BRCA family [4,5]. The clinical symptoms in patients with early stage of ovarian cancer are 
usually vague or unobvious, and over $70 \%$ of the patients at their initial diagnosis of ovarian cancer have already metastasized and shown to be in poor prognosis $[4,6]$. It is thus important to explore effective molecular screening or diagnostic markers for the early detection of ovarian cancer.

Sialyltransferases (STs) add sialic acid to the terminal site of glycolipids or glycoproteins, and play a fundamental role in post-translational modification during organ development, even during neoplastic transformation and progress $[7,8,9]$. Increasing expression of ST3Gal3 and ST6Gal1 are associated with a poor prognosis in human breast cancer [10]. ST6Gal1is involved in cell-ECM adhesion, which might contribute to metastasis of breast cancer cells as seen in our previous study [11]. Overexpression of ST3Gal4 increases the level of sialylated $\mathrm{N}$-glycans on RON receptor tyrosine kinase, which in turn activates oncogenic signaling of gastric carcinoma cells [12]. IL-1 $\beta$ increases mRNA expression of ST3Gal3/4 resulted in a modulated glycosylation pattern in pancreatic ductal adenocarcinoma cells, which then contribute to pancreatic tumor malignancy [13]. In addition, ST3Gal3 enhanced metastasis of prostate cancer, and served as a marker gene for circulating tumor cells in patients with breast cancer undergoing adjuvant therapy $[14,15]$. However, the relationship between sialyltransferases, especially ST3Gal3 and ovarian cancer progress has yet to be revealed.

Emerging data show that sialyltransferases also regulated chemosensitivity of cancer cells. Dualspecificity phosphatase 1 (DUSP1) activated p38 MAPK signaling to elevate expression of p-glycoprotein, which contributed to resistance of paclitaxel in ovarian cancer cells [16]. ST6Gal1 enhanced EGFR/ERK signaling to increase colon cancer proliferation and its tumor growth, decrease the cytotoxic effect of gefitinib [17]. Cancer stem cells (CSCs) are critical for initiation, metastasis and resistance. ST6Gal1 conferred CSCs characteristics, promoted primary ovarian cancer cells resistance to gemcitabine [18], suppressed caspase 3 activity to decreased cisplatin-induced apoptosis in several ovarian cancer cell lines [19], but the mechanism is largely unknown, although the other studies had indicated that the mechanisms of cisplatin resistance included increasing DNA damage repair and efflux of the drug [20,21]. Here we presume that other sialyltransferases might also involve in cisplatin-resistance of ovarian cancer.

We thus investigated the role of ST3Gal3 in ovarian cancer cells and found aberrant elevating expression of ST3Gal3 conferred higher cisplatin-resistance ability, which suppressed cisplatin-induced activation of caspase 8/3. Interestingly, low dose cisplatin elevated expression of ST3Gal3, while ST3Gal3 knockdown enhanced cisplatin-induced apoptosis of ovarian cancer cells. Thereby, we presented a new mechanism of cisplatin-resistance in ovarian cancer, which provided a new insight into cisplatin treatment for ovarian cancer.

\section{Materials and Methods}

\subsection{Human ovarian carcinoma Cell lines}

The human ovarian cell lines, OVCAR3 and SKOV3, were purchased from ATCC (Manassas, VA), and HO8910 and HO8910PM were purchased from the Type Culture Collection Center of Chinese Academic of Science (Shanghai, China). HO8910 was isolated from the ovary patient with malignant papillary serous adenocarcinoma, then HO8910PM was established as a highly metastatic cell line by limitingdilution cloning of the HO8910. SKOV3 cells were cultured in RPMI- 1640 medium (GIBCO, USA) containing $10 \%$ fetal bovine serum (GIBCO, USA) and 1\% penicillin-streptomycin (100X, GIBCO, USA). OVCAR3, HO8910 and HO8910PM were cultured in DMEM high-glucose medium (GIBCO, USA) 
containing $10 \%$ fetal bovine serum and $1 \%$ penicillin-streptomycin. All cell lines were cultured at $37{ }^{\circ} \mathrm{C}$ with the presence of $5 \% \mathrm{CO}_{2}$.

\subsection{RNA isolation and quantitative RT-PCR}

Total RNA was extracted using Trizol reagent (Invitrogen, USA) according to the manufacturer's instructions. Total RNA was reversely transcribed using PrimerScript ${ }^{\mathrm{TM}}$ RT Master Mix kit (TaKaRa, Dalian, China). cDNA was amplified using SYBR ${ }^{\circledR}$ Premix Ex Taq $^{\text {TM }}$ (Tli RNaseH Plus), ROX plus kit (TaKaRa, Dalian, China). Primers used in this study are shown as follow: ST3Gal1-forward (5'- TCCCGGTTCCTGCAAAGATC3') , reverse (5'-ACAAGTCCACCTCATCGCAG-3'); ST3Gal3-forward (5'-AAAACGACACTGCGCATCAC-3') reverse (5'-TCGAGTGGCCACAGATTTCC-3'); ST3Gal4-forward (5'-GCCTGCTGAATTAGCCACCAA-3'), reverse (5'-CTGGCTATGAGGGTGACGTG-3');ST6Gal1-forward (5'-AAGCTACCAGGACGAGGAGT-3'), reverse (5'GTGCAGGCACTATCGAAGAA-3'); GAPDH -forward (5'-AGCCTCAAGATCATCAGC-3'), reverse (5'GAGTCCTTCCACGATACC-3'). The PCR cycling condition was set as follow: pre-denaturation at $95{ }^{\circ} \mathrm{C}$ for 30 seconds followed by 40 cycles of $95{ }^{\circ} \mathrm{C}$ for 5 seconds, $60{ }^{\circ} \mathrm{C}$ for 40 seconds. Relative levels of mRNA expression for the target genes were normalized by comparing to GAPDH, calculated using the $2-\Delta \Delta \mathrm{Ct}$ method.

\subsection{Cell counting kit-8 assays}

SKOV3 and HO8910PM cells were seeded into a 96-well plate at a density of $5 \times 10^{3}$ cells/well, respectively. The next day, the cells were treated with various concentrations of cisplatin (Sigma, St. Louis, MO), PBS was used as negative control. 48 hours after the treatment, the cellular vitality for each of the wells was measured using cell counting kit-8 (Beyotime, Shanghai, China) according to the manufacturer's instructions. The cytotoxicity $(\%)=[1-(\mathrm{OD}$ from tested cells)/OD from control cells $]$ $* 100 \%$.

\subsection{Small interfering RNA (siRNA) transfection and cisplatin treatments}

Three pairs ST3Gal3 siRNA were designed and synthesized by Ribobio (Guangzhou, China). The sequences of ST3Gal3 siRNA are shown as follow: \#1-sense (5'-CGTGGAAGCTACACTTACT-3'), \#2-sense (5'-CCTGAATCTGGACTCTAAA-3'), \#3-sense (5'-CCTGGACGCACAATATCCA-3'). Briefly, cells were seeded into a 6 -well plate at a density of $2 \times 10^{5}$ cells/well. The next day, $7.5 \mu$ l Lipofectamine ${ }^{\circledR}$ RNAiMAX reagent (Invitrogen, USA) was diluted in $125 \mu$ Opti-MEM $^{\circledR}$ Medium (Invitrogen, USA), and 30 pmol siRNA in 125 $\mu$ Opti-MEM ${ }^{\circledast}$ Medium, mixed by vortexing respectively for 10 seconds. Then the diluted siRNA was added to the diluted Lipofectamine ${ }^{\circledR}$ RNAiMAX Reagent. The mixture was then incubated for 10 minutes at room temperature. During the incubation, the seeded cells were washed once with $3 \mathrm{ml}$ of PBS, then added with $2 \mathrm{ml}$ of fresh growth medium. After the incubation, $250 \mu$ l transfection mixture was dropped onto cells in the 6-well plate and incubated. The transfected cells were cultured for two days, and with cisplatin treatments for another 48 hours.

\subsection{Western blot assay}

Cells were collected and lysed on ice by RIPA lysis buffer (Beyotime, China) containing 1\% PMSF. Protein concentration was determined using BCA protein assay kit (Beyotime, China). $50 \mu \mathrm{g}$ of total lysate protein sample was separated by SDS-PAGE and transferred onto the PVDF membrane (BIO-RAD, USA). After blocking, membranes were incubated overnight at $4{ }^{\circ} \mathrm{C}$ with primary antibodies. Membranes were then incubated with secondary antibody for 1 hour at room temperature, and results were 
acquired using the Gel Logic 1500 imaging system (Eastman Kodak, USA). The primary antibodies were used as follow: anti-ST3Gal3 (Santa Cruz, USA), anti-caspase8 (Cell Signaling, USA), anti-caspase3 (Cell Signaling, USA), or anti-GAPDH (Beyotime, China). Anti-rabbit or anti-mouse secondary antibody (Beyotime, China) was used. GAPDH was used as internal standards. ImageJ software (http://rsb.info.nih.gov/ij/download.html) was used to quantify the immunoreactive bands, and the mean intensity of the first band was set to 1 .

\subsection{Flow Cytometry Analysis}

Cells were digested and collected by centrifugation at $1000 \mathrm{r} / \mathrm{min}$ for $5 \mathrm{~min}$. The cells were resuspended and washed briefly in $1 \mathrm{ml}$ PBS twice, before resuspending the pellets in $500 \mu \mathrm{l}$ of $1 \mathrm{X}$ binding buffer.5 $\mu$ l of Annexin-V-FITC (BD Biosciences, USA) was added into the tubes and incubated at room temperature for 15 minutes in the dark, then $5 \mu$ of propidium iodide (BD Biosciences, USA) was added and incubated at room temperature for 5 minutes in the dark. Finally, Apoptosis was immediately quantified using the FACSCanto II flow cytometer (BD Biosciences, USA) according to the manufacturer's instructions.

\subsection{Statistical analysis}

Numerical data were expressed as mean \pm S.D., and statistical analyses were performed using oneway ANOVA and Tukey-Kramer multiple comparison test (GraphPad Software Inc., La Jolla, CA). $p<0.05$ was considered to be statistically significant. Experiments were independently performed at triplicated or more, and results were qualitatively identical. Representative experiments are shown.

\section{Results}

3.1 The relationship between expression of sialyltransferases and cisplatin-induced cytotoxicity in ovarian cancer cell lines.

To investigate the expression of sialyltransferases in ovarian cancer cell lines, we performed quantitative RT-PCR assays. The mRNA expression of ST3Gal3 differed significantly among ovarian cancer cell lines, the mRNA of ST3Gal1 and ST3Gal4 were expressed significantly more in $\mathrm{HO} 8910$ and HO8910PM cells than in SKOV3 and OVCAR3 cells, but there was not significantly different between HO8910 and HO8910PM cells (Fig. 1A). The mRNA of ST6Gal1 was expressed significantly more in SKOV3 cells than in HO8910 and OVCAR3 cells, but there was not significantly different between SKOV3 and H08910PM cells. Interestingly, the mRNA of ST3Gal3 was dramatically elevated more in H08910PM cells than the others', following by OVCAR3 cells, and there was not significantly different between SKOV3 and HO8910 cells (Fig. 1A). The aberrantly expression of ST3Gal3 in ovarian cancer cells prompted us to investigate the relationship between expression of ST3Gal3 and cisplatin-resistance. Cisplatin treatment ranging from $0 \mu \mathrm{M}$ to $40 \mu \mathrm{M}$ dose-dependently induced cell death in SKOV3, HO8910 and HO8910PM cells. The $\mathrm{IC}_{50} \mathrm{~S}$ (50\% Cytotoxicity Concentration) of cisplatin in SKOV3, HO8910 and HO8910PM cells are $9 \mu \mathrm{M}, 8 \mu \mathrm{M}$ and $32 \mu \mathrm{M}$ respectively. Our data indicated that the cisplatin-resistance ability of HO8910PM cells was almost 4-fold higher than SKOV3 cells' or H08910 cells'. Collectively, aberrantly elevated expression of ST3Gal3, but not ST3Gal1, ST3Gal4 or ST6Gal1 might confer cisplatin-resistance in ovarian cancer. 


\subsection{Low dose cisplatin upregulated ST3Gal3 expression in SKOV3 cells and HO8910 cells.}

Chemoresistance is a common outcome after anti-cancer chemotherapy. To further uncovered the possible mechanism of cisplatin-resistance in ovarian cancer, according to what we found as above, we then investigated effect of cisplatin on ST3Gal3 expression in ovarian cancer cells. SKOV3 cells were incubated with various concentrations of cisplatin and total RNA was isolated and submitted to quantitative RT-PCR at each indicated time point. Interestingly, our results showed that $1 \mu \mathrm{M}$ cisplatin treatment significantly upregulated mRNA expression of ST3Gal3 within 72 hours. After 48 hours treatment, $5 \mu \mathrm{M}$ cisplatin also significantly upregulated mRNA expression of ST3Gal3, but higher dose $(20 \mu \mathrm{M})$ cisplatin treatment significantly suppressed mRNA expression of ST3Gal3 within 72 hours (Fig. $2 \mathrm{~A})$, probably due to its high cytotoxicity. After 48 hours treatment, cisplatin dose-dependently elevated ST3Gal3 protein level in SKOV3 and HO8910 cells (Fig. 2B and C). Therefore, upregulation of ST3Gal3 by low dose cisplatin seems to be a common phenomenon in ovarian cancer cell lines which have a low ST3Gal3 expression background.

\subsection{ST3Gal3 knockdown facilitated ovarian cancer cells to cisplatin-induced apoptosis.}

To further confirm the role of ST3Gal3 in cisplatin resistance, we first applied siRNA to knockdown ST3Gal3 expression in ovarian cancer cells. All three ST3Gal3 siRNAs significantly downregulated mRNA expression of ST3Gal3 in SKOV3 cells. Especially, the 1\#-siRNA decreased about 84\% mRNA expression of ST3Gal3 (Fig. 3A). Knockdown efficiency of 1\#-siRNA of ST3Gal3 was further confirmed by western blot in SKOV3 cells (Fig. 3B). We next determined whether the ST3Gal3 knockdown facilitated ovarian cancer cells to cisplatin-induced apoptosis. Our results showed that ST3Gal3 knockdown significantly increased apoptosis of SKOV3 and HO8910PM cells as compared to each siRNA of control group $\left(p^{*}<0.05\right.$ for SKOV3 cells, $p^{++}<0.01$ for HO8910PM cells), besides, ST3Gal3 knockdown significantly increased more apoptosis in HO8910PM cells than in SKOV3 cells $\left(p^{\psi}<0.05\right) .5 \mu \mathrm{M}$ cisplatin also significantly induced apoptosis of SKOV3 and HO8910PM cells while compared to each siRNA of control group $\left(p^{* *}<0.01\right.$ for SKOV3 cells, $p^{+}<0.05$ for HO8910PM cells), but there is no significant difference of cisplatin-induced apoptosis between HO8910PM cells and SKOV3 cells $\left(p^{\psi}>0.05\right)$, although cisplatin induced more apoptosis in SKOV3 cells than in HO8910PM cells. Strikingly, the combination of cisplatin and ST3Gal3 knockdown significantly and dramatically induced apoptosis of SKOV3 and HO8910PM cells while compared to each siRNA of control group ( $p^{*}<0.05$ for SKOV3 cells, $p^{++}<0.01$ for HO8910PM cells), besides, the combination of cisplatin and ST3Gal3 knockdown significantly induced more apoptosis in H08910PM cells than in SKOV3 cells ( $p^{\psi \psi}<0.01$ ) (Fig. 3C and D). Thus ST3Gal3 knockdown increased the chemosensitivity of SKOV3 and HO8910PM cells to cisplatin.

\subsection{Caspase 8/3 involved in ST3Gal3knockdown and cisplatin-induced apoptosis.}

To determine whether the caspase signaling was activated during ST3Gal3 knockdown and cisplatin induced apoptosis, we investigated the effects of ST3Gal3 knockdown and cisplatin treatments on expression of caspase3 and caspase 8. Our data showed that neither ST3Gal3 knockdown nor cisplatin had any effect on expression of caspase 3 and caspase 8. The combination of cisplatin and ST3Gal3 knockdown still had barely effect on expression of caspase 8, but it increased 1.3-fold more ST3Gal3 protein expression compared to control group (Fig.4A). Similarly, cisplatin also slightly elevated ST3Gal3 protein level in HO8910PM cells which have a high ST3Gal3 expression background. Moreover, cisplatin elevated 1.5-fold caspase3 protein level in HO8910PM cells, but not caspase 8. Interestingly, ST3Gal3 
knockdown elevated 1.3-fold caspase 8 protein level and elevated 2.1-fold caspase 3 protein level in HO8910PM cells. The combination of cisplatin and ST3Gal3 knockdown triggered caspase signaling more effective in HO8910PM cells, which elevated 1.5-fold caspase 8 protein level and elevated 2.5-fold caspase 3 protein level (Fig. 4B). Thus ST3Gal3 knockdown enhanced cisplatin-induced apoptosis via facilitating activation of caspase signaling.

\section{Discussion}

Despite increasing survival rates, chemoresistance and recurrence remain the major obstacles to clinic chemotherapeutic of ovarian cancer. Clinic data indicated that AKT inhibition might reverse platinum resistance in ovarian cancer [22]. Interestingly, in platinum-sensitive ovarian cancer cells, cisplatin in combination with the MEK1/2 inhibitor synergistically enhanced apoptosis, but exhibited increased phospho-ERK1/2, down-regulation of apoptosis-related factors (BAX, PUMA, FOXO1) and of phosphatases inhibiting ERK1/2 (DUSP5, DUSP6) [23]. Correctively, these data indicated that cisplatin resistance was a complex context, and might be largely diversification in activation of dependent/nondependent MAPK/AKT, MAPK/ERK signaling. Sialyltransferases serve as an upstream signaling regulators to sialylate glycolipids, glycoproteins and receptors like EGFRs, then trigger outside-in axis signaling which play an important role in chemoresistance [24-26]. However, upstream pathways of cisplatin resistance largely remain elusive, which prompt us to investigate the relationship between sialyltransferases and cisplatin resistance in ovarian cancer.

We first examined several sialyltransferases expression in ovarian cancer cell lines. We found that the mRNA expression pattern of sialyltransferases varied among cell lines. Only ST3Gal3 differed significantly between HO8910 and HO8910PM cells, and the mRNA expression of ST3Gal3 was dramatically and significantly elevated more in HO8910PM cells than the other ovarian cancer cells. It's reported that ST3Gal3 sialylated $\alpha 2 \beta 1$ integrin receptors to result in bone metastasis of LNCAPderivative C4-2B prostate cancer cells [27]. Overexpression of ST3Gal3 increased sialyl-Lewis(x) levels to enhance adhesion and metastasis of pancreatic cancer cell [28]. But the association between ST3Gal3 and cisplatin resistance has been rarely reported. Our data showed that H08910PM cells exhibit almost 4-fold more cisplatin resistance than the other ovarian cancer cells. Therefore, ST3Gal3 might not only involve in metastasis of many cancers, but also contribute to cisplatin resistance of ovarian cancer.

The mechanism for the chemoresistance of cancer cells is complicated, its predisposing factors including aberrant expression of multidrug resistance-associated proteins (MRPs), activation of drug efflux pump and existence of cancer stem cells [29-31]. In the present study, our data showed that low dose cisplatin treatment elevated the expression of ST3Gal3 as a common phenomenon in several ovarian cancer cell lines, though the mechanism remains to be further illustrated. Otherwise, ST3Gal3 knockdown significantly increased more apoptosis in HO8910PM cells (higher expression of ST3Gal3) than in SKOV3 cells (lower expression of ST3Gal3). Moreover, combined cisplatin and ST3Gal3 knockdown significantly induced more apoptosis in HO8910PM cells than in SKOV3 cells, suggests that viability of ovarian cancer cells partly if not all, relies on ST3Gal3 expression level, while ST3Gal3 knockdown facilitated ovarian cancer cells to cisplatin-induced apoptosis. Meanwhile, our data shows that ST3Gal3 knockdown effectively increased caspase 8 and caspase 3 protein in HO8910PM cells, but not in SKOV3 cells. Interestingly, low dose cisplatin elevated ST3Gal3 protein level but decreased caspase 8 protein level in SKOV3 cells, which might be due to its low expression of ST3Gal3. However, it presented a new negative regulation loop of cisplatin-induced apoptosis by ST3Gal3, which was 
confirmed by that the combination of cisplatin and ST3Gal3 knockdown dramatically and significantly induced apoptosis in ovarian cancer cells.

Taken together, we presented a new negative regulation loop of cisplatin-induced apoptosis by ST3Gal3, which would contribute to resistance of cisplatin, and provide a precaution for clinic treatment of cisplatin. There were several approaches to reverse chemoresistance, including target to cancer stem cells, inhibition of MDR pathway [32-34]. Herein, our data showed that ST3Gal3 down-regulation sensitized high cisplatin-resistant ovarian cancer cells to cisplatin, which could contribute to prevent resistance and relapse of ovarian cancer.

\section{Acknowledgments}

This work was supported by the National Science Foundation of China (81071751, 30770487), the Opening Project of Zhejiang provincial Top Key Discipline of Pharmaceutical Sciences.

\section{References}

[1] McCluggage WG, Judge MJ, Clarke BA, Davidson B, Gilks CB, Hirschowitz L, et al., International Collaboration on Cancer Reporting, International Collaboration on Cancer Reporting. Data set for reporting of ovary, fallopian tube and primary peritoneal carcinoma: recommendations from the International Collaboration on Cancer Reporting (ICCR), Mod Pathol. 28 (2015) 1101-1122.

[2] Prat J; FIGO Committee on Gynecologic Oncology, FIGO's staging classification for cancer of the ovary, fallopian tube, and peritoneum: abridged republication, J Gynecol Oncol. 26 (2015) 87-89.

[3] R.L. Siegel, K.D. Miller, A. Jemal, Cancer statistics, 2016, CA Cancer J. Clin. 66 (2016) 7-30.

[4] Cardenas C, Alvero AB, Yun B, Mor G, Redefining the origin and evolution of ovarian cancer: A hormonal connection, Endocr Relat Cancer. (2016) pii: ERC-16-0209.

[5] Gorodnova TV, Sokolenko AP, Ivantsov AO, Iyevleva AG, Suspitsin EN, Imyanitov EN, et al., High response rates to neoadjuvant platinum-based therapy in ovarian cancer patients carrying germline BRCA mutation, Cancer Lett. 369 (2015) 363-367.

[6] Ebell, MH; Culp, MB; Radke, TJ, A Systematic Review of Symptoms for the Diagnosis of Ovarian Cancer, Am J Prev Med. 50 (2016) 384-394.

[7] Wang B, Molecular mechanism underlying sialic acid as an essential nutrient for brain development and cognition, Adv Nutr. 3 (2012) 465S-472S.

[8] Vajaria BN, Patel KR, Begum R, Patel PS, Sialylation: An Avenue to Target Cancer Cells, Pathol Oncol Res. 22 (2016) 443-447.

[9] Yoo SW, Motari MG, Susuki K, Prendergast J, Mountney A, Schnaar RL, et al., Sialylation regulates brain structure and function, FASEB J. 29(2015) 3040-3053.

[10] Recchi MA1, Hebbar M, Hornez L, Harduin-Lepers A, Peyrat JP, Delannoy P, Multiplex reverse transcription polymerase chain reaction assessment of sialyltransferase expression in human breast cancer, Cancer Res. 58 (1998) 4066-4070. 
[11] Lin S, Kemmner W, Grigull S, Schlag PM, Cell surface alpha 2,6 sialylation affects adhesion of breast carcinoma cells, Exp Cell Res. 276(2002) 101-110.

[12] Mereiter S, MaGalhães A, Adamczyk B, Jin C, Almeida A, Reis CA, et al., Glycomic analysis of gastric carcinoma cells discloses glycans as modulators of RON receptor tyrosine kinase activation in cancer, Biochim Biophys Acta. 1860(2016) 1795-1808.

[13] Bassagañas S, Allende H, Cobler L, Ortiz MR, Llop E, Peracaula R, et al., Inflammatory cytokines regulate the expression of glycosyltransferases involved in the biosynthesis of tumor-associated sialylated glycans in pancreatic cancer cell lines, Cytokine. 75(2015) 197-206.

[14] Van Slambrouck S, Groux-Degroote S, Krzewinski-Recchi MA, Cazet A, Delannoy P, Steelant WF, Carbohydrate-to-carbohydrate interactions between $\alpha 2,3$-linked sialic acids on $\alpha 2$ integrin subunits and asialo-GM1 underlie the bone metastatic behaviour of LNCAP-derivative C4-2B prostate cancer cells, Biosci Rep. 34(2014). pii: e00138. doi: 10.1042/BSR20140096.

[15] Kölbl AC, Hiller RA, Ilmer M, Liesche F, Heublein S, Andergassen U, et al., Glycosyltransferases as marker genes for the quantitative polymerase chain reaction-based detection of circulating tumour cells from blood samples of patients with breast cancer undergoing adjuvant therapy, Mol Med Rep. 12(2015) 2933-2938.

[16] Kang YS, Seok HJ, Jeong EJ, Kim Y, Yun SJ, Kim JS, et al., DUSP1 induces paclitaxel resistance through the regulation of p-glycoprotein expression in human ovarian cancer cells, Biochem Biophys Res Commun. (2016) pii: S0006-291X (16)31150-0. doi: 10.1016/j.bbrc.2016.07.035.

[17] Park JJ, Yi JY, Jin YB, Lee YJ, Lee JS, Lee M, et al., Sialylation of epidermal growth factor receptor regulates receptor activity and chemosensitivity to gefitinib in colon cancer cells, Biochem Pharmacol. 83 (2012) 849-857.

[18] Schultz MJ, Holdbrooks AT, Chakraborty A, Grizzle WE, Landen CN, Bellis SL, et al., The TumorAssociated Glycosyltransferase ST6Gal-I Regulates Stem Cell Transcription Factors and Confers a Cancer Stem Cell Phenotype, Cancer Res. 76 (2016) 3978-3988.

[19] Schultz MJ, Swindall AF, Wright JW, Sztul ES, Landen CN, Bellis SL, ST6Gal-I sialyltransferase confers cisplatin resistance in ovarian tumor cells, J Ovarian Res. 6 (2013) 25. doi: 10.1186/1757-2215-6-25.

[20] Cong Y, Wang L, Wang Z, He S, Zhou D, Jing X, et al., Enhancing Therapeutic Efficacy of Cisplatin by Blocking DNA Damage Repair, ACS Med Chem Lett. 7 (2016) 924-928.

[21] Chuman Y, Chen ZS, Sumizawa T, Furukawa T, Haraguchi M, Takebayashi $Y$, et al., Characterization of the ATP-dependent LTC4 transporter in cisplatin-resistant human KB cells, Biochem Biophys Res Commun. 226 (1996) 158-165.

[22] Cheraghchi-Bashi A, Parker CA, Curry E, Salazar JF, Gungor H, Stronach EA, et al., A putative biomarker signature for clinically effective AKT inhibition: correlation of in vitro, in vivo and clinical data identifies the importance of modulation of the mTORC1 pathway, Oncotarget. 6 (2015) 4173641749. 
[23] Cossa G, Lanzi C, Cassinelli G, Carenini N, Arrighetti N, Perego P, et al., Differential outcome of MEK1/2 inhibitor-platinum combinations in platinum-sensitive and -resistant ovarian carcinoma cells, Cancer Lett. 347 (2014) 212-224.

[24] Zhang X, Dong W, Zhou H, Li H, Wang N, Jia L, et al., $\alpha$-2,8-Sialyltransferase Is Involved in the Development of Multidrug Resistance via PI3K/Akt Pathway in Human Chronic Myeloid Leukemia, IUBMB Life. 67 (2015) 77-87.

[25] Park JJ, Yi JY, Jin YB, Lee YJ, Lee JS, Lee M, et al., Sialylation of epidermal growth factor receptor regulates receptor activity and chemosensitivity to gefitinib in colon cancer cells, Biochem Pharmacol. 83 (2012) 849-857.

[26] Yen HY, Liu YC, Chen NY, Tsai CF, Wang YT, Wong CH, et al., Effect of sialylation on EGFR phosphorylation and resistance to tyrosine kinase inhibition, Proc Natl Acad Sci U S A. 112 (2015) 6955-6960.

[27] Van Slambrouck S, Groux-Degroote S, Krzewinski-Recchi MA, Cazet A, Delannoy P, Steelant WF, Carbohydrate-to-carbohydrate interactions between $\alpha 2,3$-linked sialic acids on $\alpha 2$ integrin subunits and asialo-GM1 underlie the bone metastatic behaviour of LNCAP-derivative C4-2B prostate cancer cells, Biosci Rep. 34 (2014) pii: e00138. doi: 10.1042/BSR20140096.

[28] Pérez-Garay M, Arteta B, Pagès L, de Llorens R, de Bolòs C, Peracaula R, et al., alpha2,3sialyltransferase ST3Gal III modulates pancreatic cancer cell motility and adhesion in vitro and enhances its metastatic potential in vivo, PLoS One. 5(2010). pii: e12524. doi: 10.1371/ journal.pone.0012524.

[29] Prasad R, Rawal MK, Efflux pump proteins in antifunGal resistance, Front Pharmacol. 5 (2014) 202. doi: 10.3389/fphar.2014.00202.

[30] Abdallah EA, Fanelli MF, Souza E Silva V, Machado Netto MC, Gasparini Junior JL, Chinen LT, et al., MRP1 expression in CTCs confers resistance to irinotecan-based chemotherapy in metastatic colorectal cancer, Int J Cancer. 139 (2016) 890-988.

[31] Carnero A, Garcia-Mayea Y, Mir C, Lorente J, Rubio IT, LLeonart ME, The cancer stem-cell signaling network and resistance to therapy, Cancer Treat Rev. 49 (2016) 25-36.

[32] Liu N, Mei L, Fan X, Tang C, Ji X, Wu X, et al., Phosphodiesterase 5/protein kinase G signal governs stemness of prostate cancer stem cells through Hippo pathway, Cancer Lett. 378 (2016) 38-50.

[33] Hermawan A, Wagner E, Roidl A, Consecutive salinomycin treatment reduces doxorubicin resistance of breast tumor cells by diminishing drug efflux pump expression and activity, Oncol Rep. 35 (2016) 1732-1740.

[34] Bieczynski F, Torres WD, Painefilu JC, Castro JM, Bianchi VA, Luquet CM, et al., Alterations in the intestine of Patagonian silverside (Odontesthes hatcheri) exposed to microcystin-LR: Changes in the glycosylation pattern of the intestinal wall and inhibition of multidrug resistance proteins efflux activity, Aquat Toxicol. 178 (2016) 106-117.

\section{Figure legends}


Figure 1. The relationship between expression of sialyltransferases and cisplatin-induced cytotoxicity in ovarian cancer cell lines. (A) Quantitative RT-PCR assays for ST3Gal1, ST3Gal3, ST3Gal4 and ST6Gal1 mRNA expression in ovarian cancer cell lines. (B) Cytotoxicity to SKOV3 cells, HO8910 cells and H08910PM cells treated with indicated concentrations of cisplatin for 48 hours. ${ }^{* * *} p<0.001, * * p<0.01$, ${ }^{*} p<0.05$ versus SKOV3 cells, $+++p<0.001$ versus $\mathrm{HO} 8910$ cells.

Figure 2. Low dose cisplatin upregulated ST3Gal3 expression in SKOV3 cells and HO8910 cells. (A) After indicated treatments of cisplatin, quantitative RT-PCR was applied for ST3Gal3 mRNA expression in SKOV3 cells, the mRNA level of ST3Gal3 in $0 \mu \mathrm{M}$ cisplatin is set as 1.0 at each time point. (B) After treating with indicated concentrations of cisplatin for 48 hours, then western blot analysis was performed for the protein expression of ST3Gal3 in SKOV3 cells, GAPDH served as loading control. (C) After treating with indicated concentrations of cisplatin for 48 hours, then western blot analysis was performed for the protein expression of ST3Gal3 in HO8910 cells, GAPDH served as loading control. *** $p<0.001,{ }^{* *} p<0.01,{ }^{*} p<0.05$ versus control group.

Figure 3. ST3Gal3 knockdown facilitated ovarian cancer cells to cisplatin-induced apoptosis. (A) After transfecting ST3Gal3 siRNAs into SKOV3 cells, quantitative RT-PCR was applied to verify the knockdown efficiency. (B) Western blot analysis was performed for verification the knockdown efficiency of ST3Gal3 siRNA-1\# in SKOV3 cells, GAPDH served as loading control. (C) After indicated treatments, the apoptosis was analyzed in SKOV3 cells and HO8910 cells. (D) Quantification of apoptosis from (C). ${ }^{* * *} p<0.001,{ }^{* *}$ $p<0.01,{ }^{*} p<0.05$ versus SKOV3 control group, $+++p<0.001$ versus HO8910PM control group, ${ }^{\psi \psi} p<0.01$, ${ }^{\Psi} p<0.05$ the same treatment between SKOV3 cells and HO8910PM cells.

Figure 4. ST3Gal3 knockdown facilitated ovarian cancer cells to cisplatin-induced apoptosis. (A) After indicated treatments, then western blot analysis was performed for the protein expression of ST3Gal3, caspase 3 and caspase 8 in SKOV3 cells, GAPDH served as loading control. (B) After indicated treatments, then western blot analysis was performed for the protein expression of ST3Gal3, caspase 3 and caspase 8 in HO8910PM cells, GAPDH served as loading control. 
Alpha2,3-sialyltransferase III knockdown sensitized ovarian cancer cells to cisplatin-induced apoptosis 
1. The relationship between expression of sialyltransferases and cisplatin-induced cytotoxicity in ovarian cancer cell lines.

A

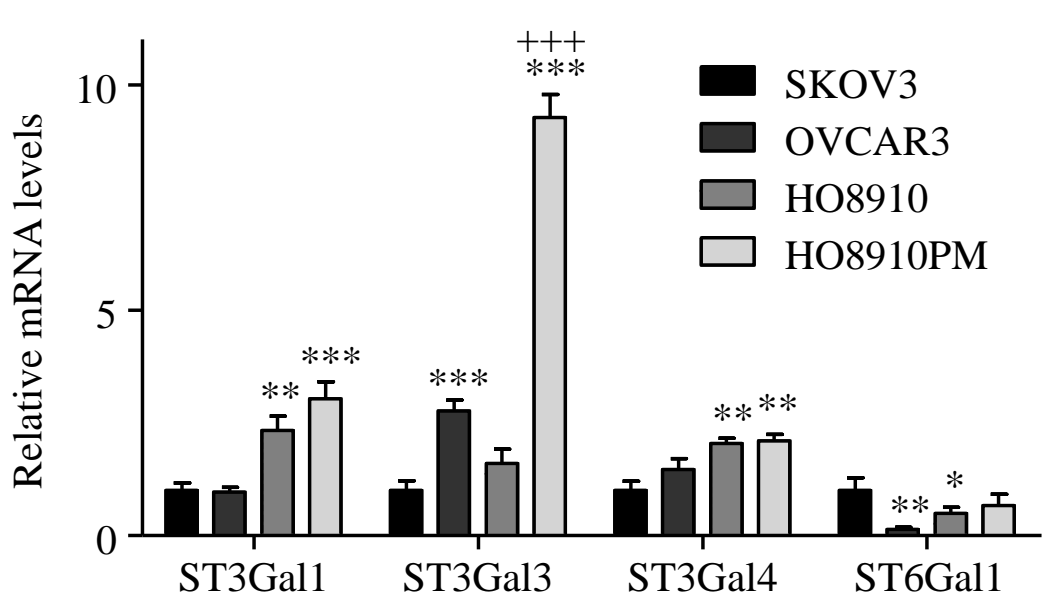

B

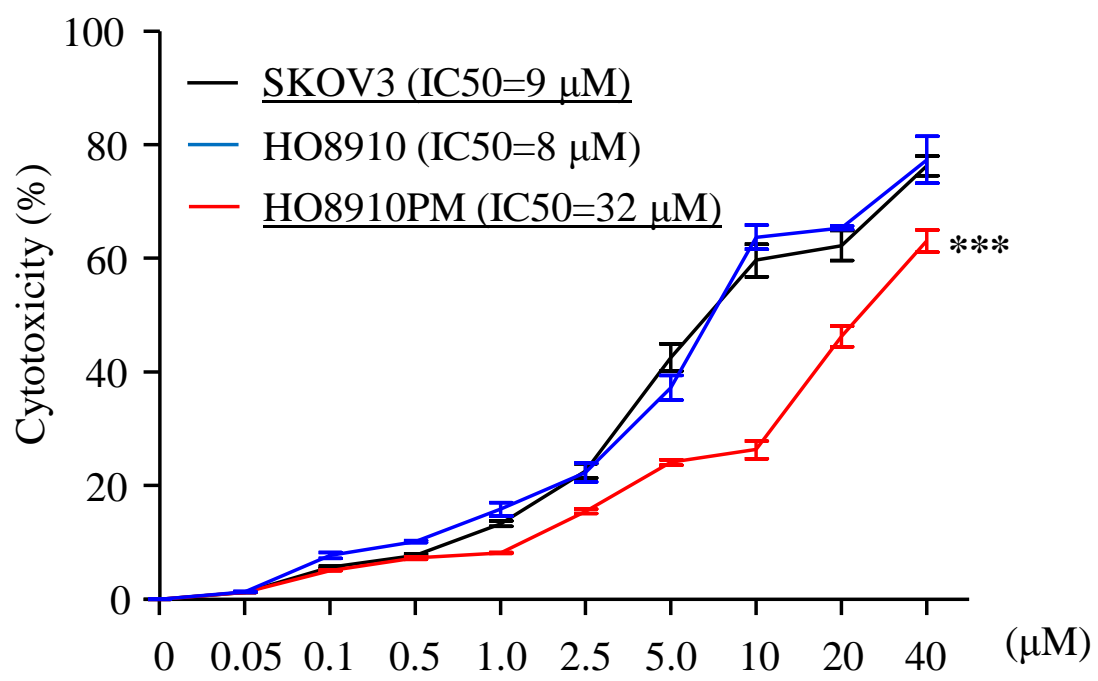




\section{Low dose cisplatin upregulated ST3Gal3 expression in SKOV3 cells and HO8910 cells.}

A

ST3Gal3 mRNA of SKOV3

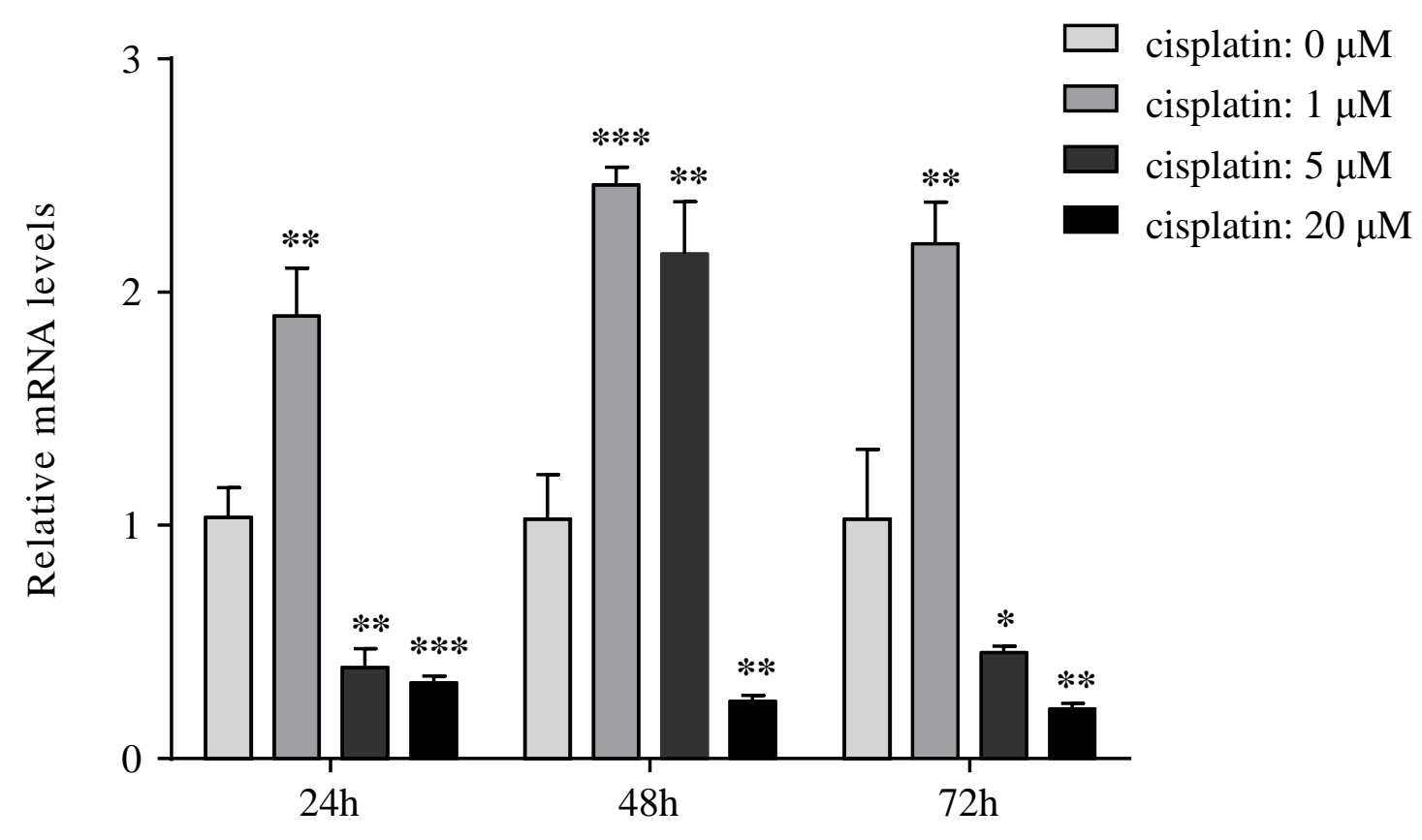

\section{B}

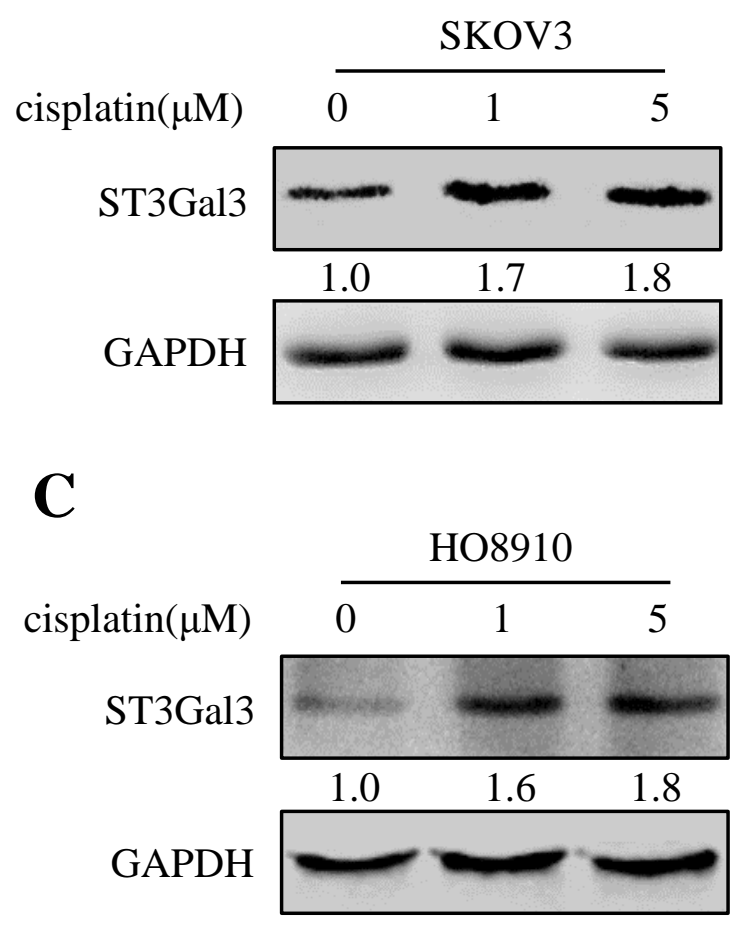




\section{ST3Gal3 knockdown facilitated ovarian cancer cells to cisplatin-induced apoptosis.}

A

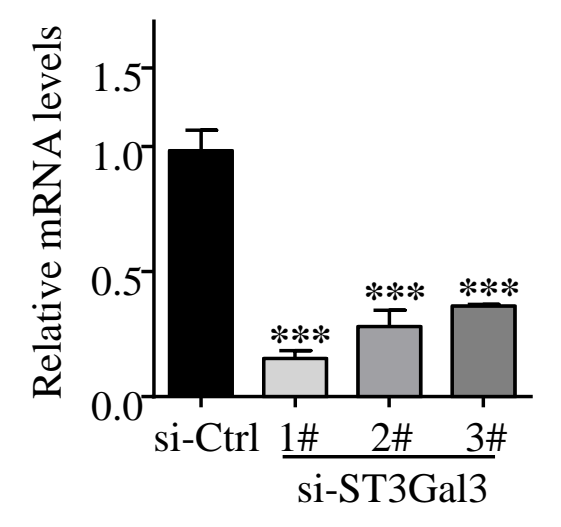

B

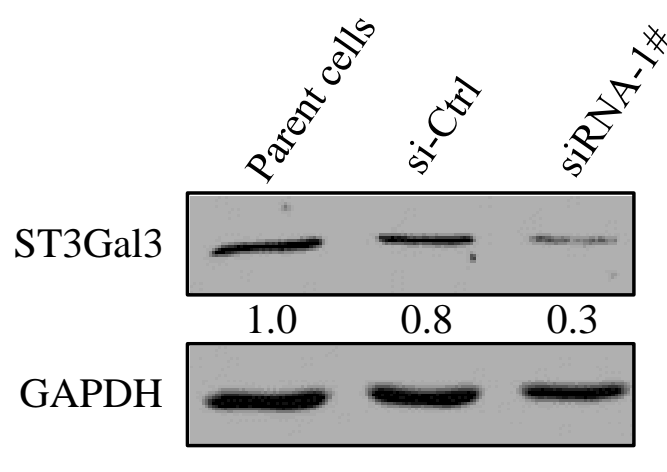

C
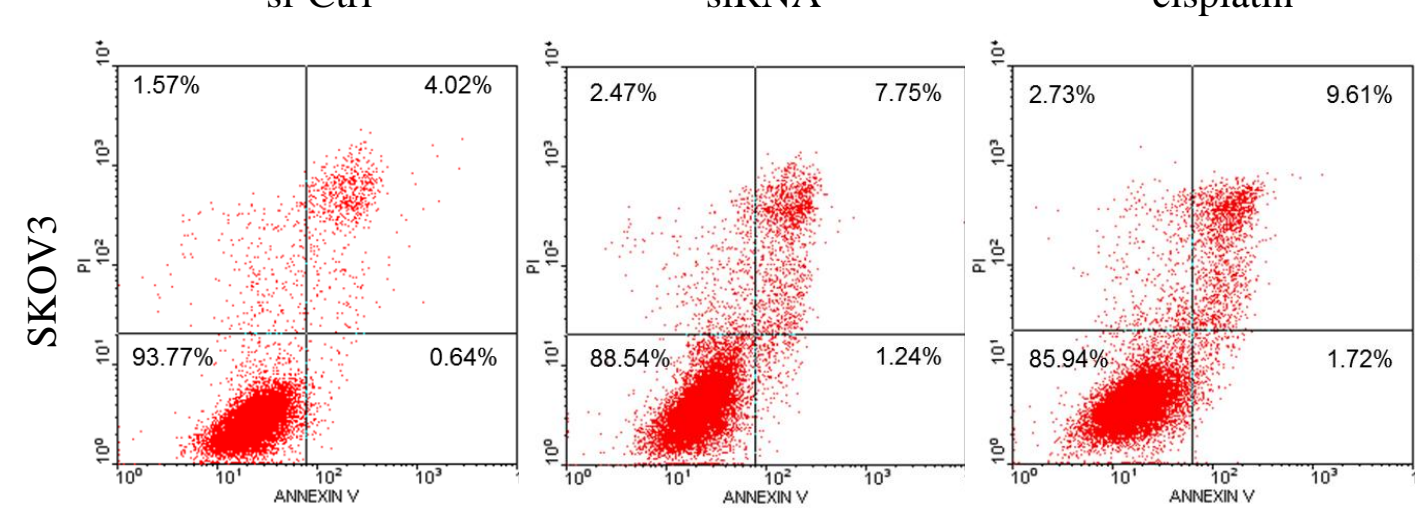
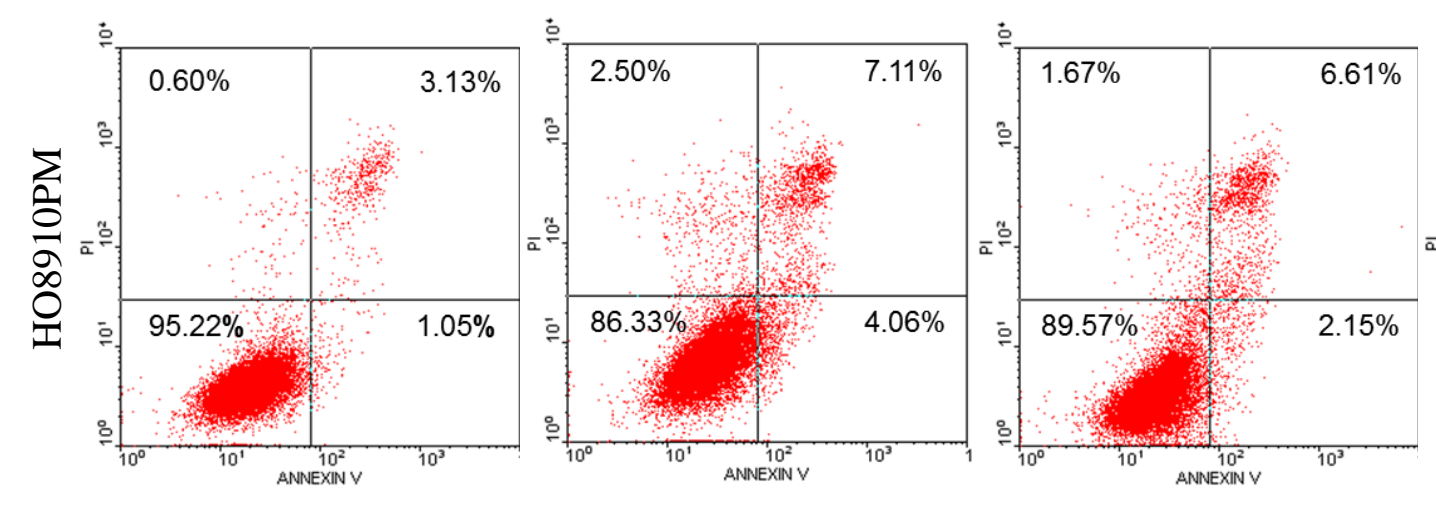

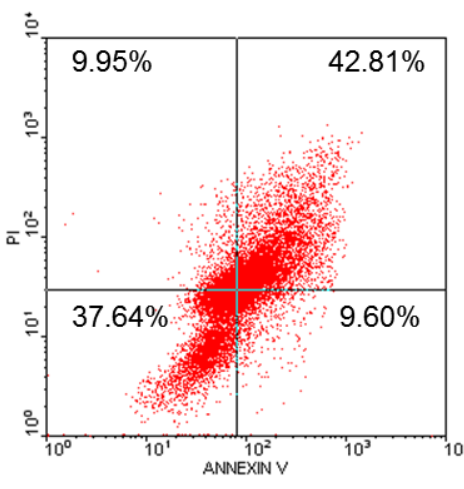

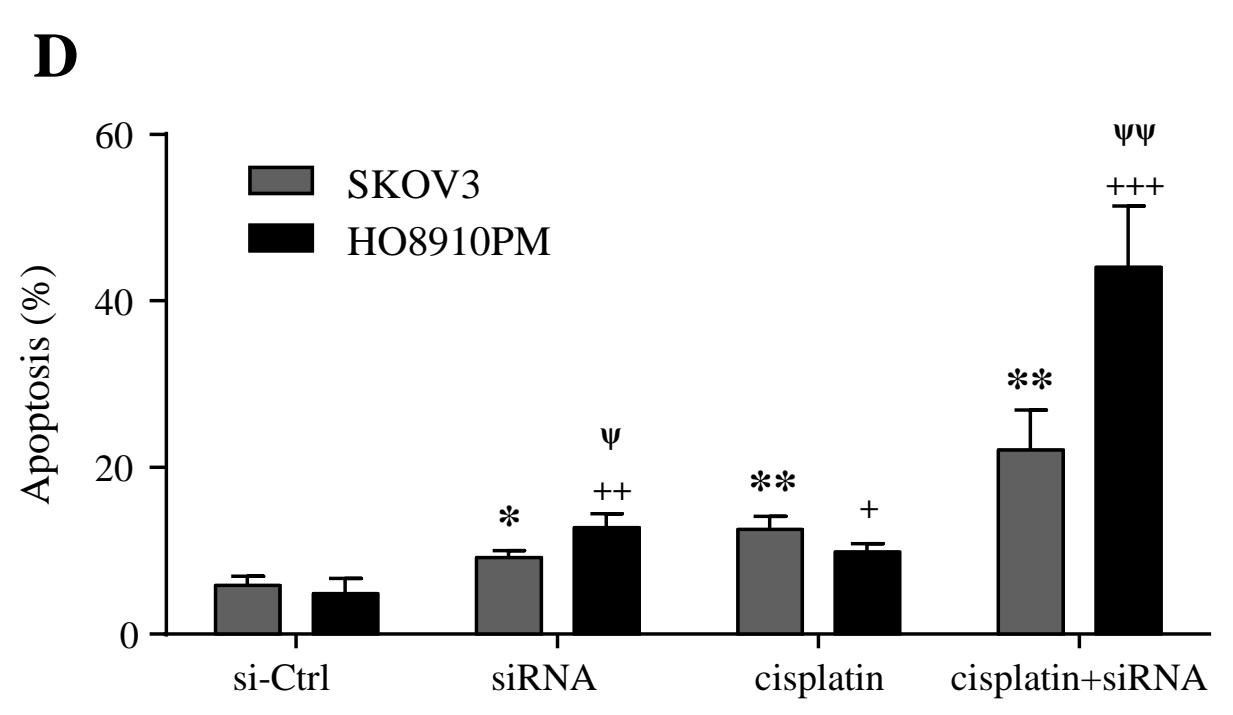

cisplatin+siRNA

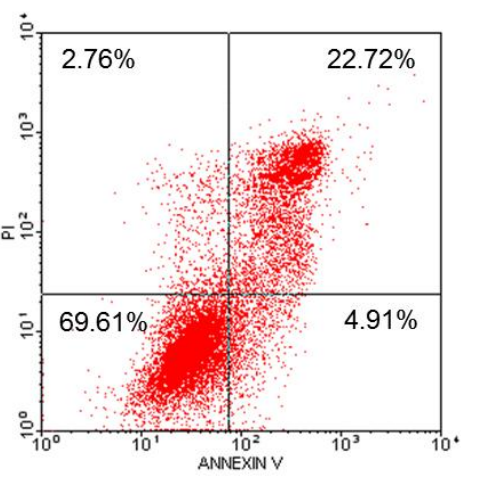

ANNEXIN Y 


\section{Caspase 8/3 involved in ST3Gal3 knockdown and cisplatin-induced apoptosis.}

A

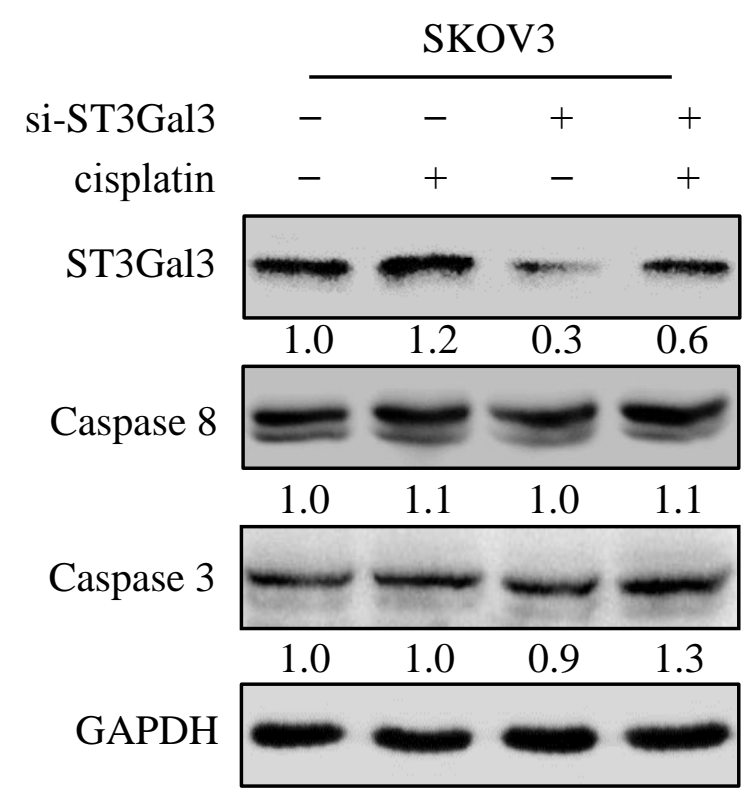

B

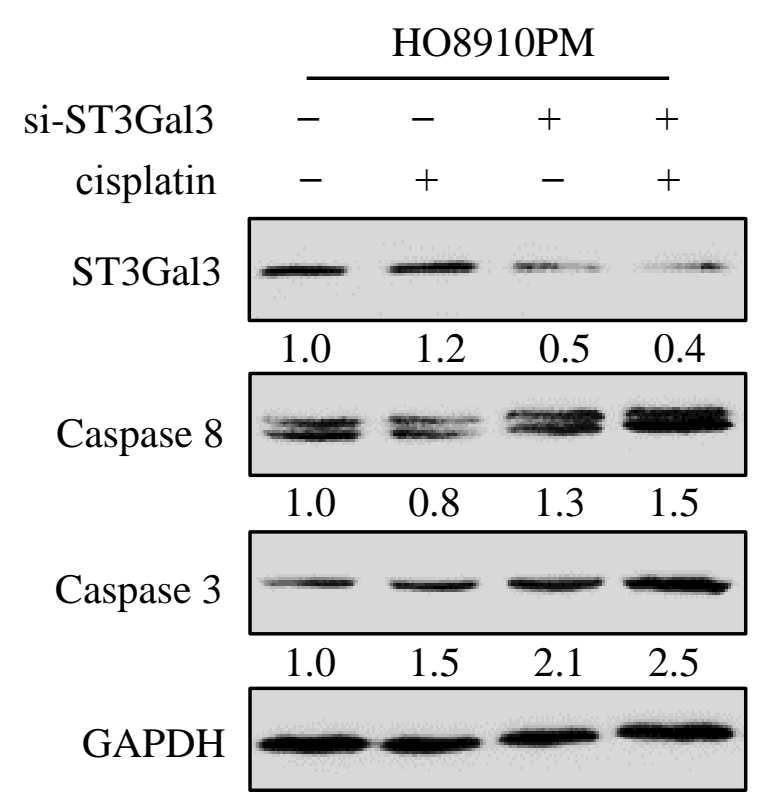

